\title{
Host-microbiome interactions in human type 2 diabetes following prebiotic fibre (galacto-oligosaccharide) intake
}

\author{
Camilla Pedersen $^{1}$, Edith Gallagher ${ }^{2}$, Felicity Horton ${ }^{2}$, Richard J. Ellis ${ }^{3}$, Umer Z. Ijaz ${ }^{4}$, Huihai Wu ${ }^{1}$, \\ Etana Jaiyeola ${ }^{1}$, Onyinye Diribe ${ }^{1}$, Thibaut Duparc ${ }^{5}$, Patrice D. Cani ${ }^{5}$, Glenn R. Gibson ${ }^{6}$, Paul Hinton ${ }^{2}$, \\ John Wright ${ }^{1,7}$, Roberto La Ragione ${ }^{1}$ and M. Denise Robertson ${ }^{1 *}$ \\ ${ }^{1}$ Faculty of Health and Medical Sciences, University of Surrey, Guildford GU2 $7 X H$, UK \\ ${ }^{2}$ Medical Physics - Nuclear Medicine, Royal Surrey County Hospital, Guildford GU2 7XX, UK \\ ${ }^{3}$ Animal and Plant Health Agency, Addlestone KT15 3NB, UK \\ ${ }^{4}$ School of Engineering, University of Glasgow, Glasgow G12 8LT, UK \\ ${ }^{5}$ Louvain Drug Research Institute, Catholic University of Louvain, Brussels B-1200, Belgium \\ ${ }^{6}$ Department of Food and Nutritional Sciences, University of Reading, Reading RG6 6AP, UK \\ ${ }^{7}$ CEDAR Centre, Royal Surrey County Hospital, Guildford GU2 7XX, UK \\ (Submitted 6 May 2016 - Final revision received 26 October 2016 - Accepted 27 October 2016 - First published online 15 December 2016)
}

\section{Abstract}

Aberrant microbiota composition and function have been linked to several pathologies, including type 2 diabetes. In animal models, prebiotics induce favourable changes in the intestinal microbiota, intestinal permeability (IP) and endotoxaemia, which are linked to concurrent improvement in glucose tolerance. This is the first study to investigate the link between IP, glucose tolerance and intestinal bacteria in human type 2 diabetes. In all, twenty-nine men with well-controlled type 2 diabetes were randomised to a prebiotic (galacto-oligosaccharide mixture) or placebo (maltodextrin) supplement $(5.5 \mathrm{~g} / \mathrm{d}$ for 12 weeks). Intestinal microbial community structure, IP, endotoxaemia, inflammatory markers and glucose tolerance were assessed at baseline and post intervention. IP was estimated by the urinary recovery of oral ${ }^{51} \mathrm{Cr}-\mathrm{EDTA}$ and glucose tolerance by insulin-modified intravenous glucose tolerance test. Intestinal microbial community analysis was performed by highthroughput next-generation sequencing of $16 \mathrm{~S}$ rRNA amplicons and quantitative PCR. Prebiotic fibre supplementation had no significant effects on clinical outcomes or bacterial abundances compared with placebo; however, changes in the bacterial family Veillonellaceae correlated inversely with changes in glucose response and IL-6 levels $(r-0.90, P=0.042$ for both) following prebiotic intake. The absence of significant changes to the microbial community structure at a prebiotic dosage/length of supplementation shown to be effective in healthy individuals is an important finding. We propose that concurrent metformin treatment and the high heterogeneity of human type 2 diabetes may have played a significant role. The current study does not provide evidence for the role of prebiotics in the treatment of type 2 diabetes.

Key words: Prebiotic supplements: Diabetes: Gut microbiota: Intestinal permeability: Endotoxaemia

Evidence from animal studies supports a causal link between low-grade inflammation, insulin resistance and impaired intestinal barrier function ${ }^{(1,2)}$; however, we recently demonstrated for the first time that intestinal permeability (IP) is compromised in type 2 diabetes (T2D) patients compared with healthy age- and BMI-matched volunteers ${ }^{(3)}$. Increased small IP as measured by urinary excretion of orally administered ${ }^{51} \mathrm{Cr}$-EDTA was significantly and positively correlated with the inflammatory marker TNF- $\alpha$. This may indicate that the chronic systemic low-grade inflammation characterising metabolic diseases such as T2D is associated with a leaky gut in humans.
It is hypothesised that the impaired intestinal barrier leads to an increased translocation of the gram-negative bacteria cell membrane component lipopolysaccharide (LPS) (as well as whole bacteria and other luminal antigens) into the circulation, which results in metabolic endotoxaemia. LPS is a ligand of the toll-like receptor 4 (TLR-4). Activation of TLR- 4 signalling by LPS results in a low-grade inflammation, which affects insulin signalling and thus induces insulin resistance ${ }^{(1)}$. Interestingly circulating LPS is indeed elevated in T2D compared with healthy controls ${ }^{(4,5)}$. However, whether this is due to increased paracellular movement or due to fat-induced LPS absorption through increased chylomicron formation is unclear ${ }^{(6)}$.

Abbreviations: GOS, galacto-oligosaccharide; IP, intestinal permeability; LPS, lipopolysaccharide; qPCR, quantitative real-time PCR; T2D, type 2 diabetes.

* Corresponding author: Dr M. D. Robertson, fax +441483688 501, email m.robertson@surrey.ac.uk 
Intestinal dysbiosis in T2D has been observed in a number of cross-sectional studies ${ }^{(7-12)}$. Larsen et $a l^{(7)}$ found that Betaproteobacteria and the Bacteroidetes:Firmicutes ratio correlated positively with plasma glucose concentrations. Thus, as a potential therapeutic target, altering intestinal bacterial community structure and thereby reducing LPS load and uptake, may be beneficial in T2D. An approach to changing the intestinal bacterial composition by diet is with the use of prebiotics and probiotics. Studies in rodents suggest that prebiotics, probiotics and synbiotics may improve intestinal barrier function and glucose control $^{(2,13-15)}$. However, few studies have investigated the use of prebiotic supplementation in human $\mathrm{T}_{2} \mathrm{D}^{(16-22)}$ and none in terms of the potential mechanistic effects on the intestinal barrier. This is the first study to investigate the effects of prebiotic supplementation on intestinal bacteria, IP, endotoxaemia and glucose tolerance concurrently in T2D patients.

\section{Methods}

This was a randomised, double-blind, placebo-controlled parallel study comparing the effects of prebiotic supplementation with placebo treatment for 12 weeks on glucose control, IP, intestinal bacterial composition, endotoxaemia and inflammatory markers in patients with T2D. The protocol was approved by the Central London NRES Committee (REC reference no. 11/LO/1141) and the University of Surrey Ethics Committee and was conducted according to the declaration of Helsinki. The trial was registered at the UK Clinical Research Network portfolio database under trial identifier ISRCTN07813749.

\section{Subjects}

Men with well-controlled T2D aged 42-65 years were recruited through local general practices and advertisement in a local newspaper. Because of the repeated administration of the radioactive compound ${ }^{51} \mathrm{Cr}$-EDTA and the potential influence of the menstrual cycle on outcomes, women were excluded from the study. All patients provided written informed consent. Exclusion criteria included the use of antibiotics in the past 3 months, use of anti-inflammatory medications (except a lowdose $(75 \mathrm{mg} / \mathrm{d})$ aspirin), diuretics, proton-pump inhibitors, inflammatory bowel disease, Crohn's disease, coeliac disease and irritable bowel syndrome. Patients were asked to exclude probiotic products and prebiotic supplements (other than the study supplement) from their diet for 2 weeks before the first study visit and throughout the study. Furthermore, they were asked not to change their lifestyle during the study. The sample size for this study was based on the primary outcome measure of changes to IP and based on our own published pilot data using this method in patients with well-controlled $\mathrm{T}_{2} \mathrm{D}^{(3)}$. In this parallel-design study, thirty patients provided $80 \%$ power to detect a treatment difference between groups of $1.6 \%$ in total permeability, using the calculated SD in this cohort of $1.57(\alpha 0.05)$.

\section{Study protocol}

After the screening procedure, patients were randomised to either prebiotic fibre (galacto-oligosaccharide mixture (GOS mixture); $\mathrm{Bi}^{2}$ muno) or placebo (maltodextrin) supplementation for 12 weeks according to a randomisation scheme generated at randomization.com. Both supplements were supplied by Clasado Ltd as dry white powders in sachets each containing $5.5 \mathrm{~g}$ and were readily mixed into beverages or food. The GOS mixture has been used in previous trials and is described by Vulevic et al. ${ }^{(23)}$. A dose of $5.5 \mathrm{~g}$ of GOS mixture has previously been demonstrated to have a bifidogenic effect in healthy individuals of this age and BMI, and be well tolerated in terms of gastrointestinal effects ${ }^{(23,24)}$. Patients were contacted twice during the 12-week supplementation to monitor side effects and compliance. Patients returned unused sachets following the supplementation to verify compliance. Dietary intake data (7-d diet diary), clinical data and faecal samples were collected at baseline and at the end of the intervention. The diet diaries were analysed in DietPlan6 (Forestfield Software Ltd). Faecal samples were collected in sterile universal polystyrene containers and were kept refrigerated. Faecal samples were stored at $-20^{\circ} \mathrm{C}$ initially and in a $-80^{\circ} \mathrm{C}$ freezer for long-term storage.

The co-primary outcomes of the study were changes in IP, endotoxaemia and glucose tolerance. Secondary outcomes were changes in intestinal bacterial composition, inflammatory markers, lipids, blood pressure and anthropometric measurements. Use of metformin was considered a confounding factor. However, as thirteen out of fourteen patients in the prebiotic group were metformin treated, it was not possible to perform a subgroup analysis to explore a potential interaction between metformin and prebiotic treatment.

\section{Intestinal permeability}

IP was measured by 24-h urinary excretion of orally administered ${ }^{51}$ Cr-EDTA, as previously described ${ }^{(3)}$. We utilised ${ }^{51} \mathrm{Cr}$-EDTA as a probe, as it is stable in the colonic luminal environment allowing assessment of colonic permeability and it is easily detected in the urine ${ }^{(25)}$.

\section{Anthropometric and blood pressure measurements}

Having fasted overnight, patients attended the CEDAR Centre of the Royal Surrey County Hospital. Body weight and body composition were measured by bioimpedance (Tanita). Waist circumference was measured at the level of the navel with a tape measure. Blood pressure was measured on the nondominant arm after $5 \mathrm{~min}$ of rest in a semi-upright position, and the mean of three readings was calculated (Omron MX3 Plus; Omron Healthcare Europe).

\section{Glucose tolerance, inflammatory markers and lipids}

Glucose tolerance was assessed using a frequently sampled insulin-modified intravenous glucose tolerance test (IVGTT), as previously described $^{(26)}$. Blood was collected in EDTA tubes for glucose, insulin and C-peptide and HbA1c measurements and into serum tubes containing clotting activator or pyrogen-free tubes for measurements of inflammatory markers, lipids and LPS in serum. Aprotinin was added to blood samples (200 kallikrein inhibiting units/ml blood) collected for C-peptide 
measurement. Blood samples were centrifuged at $3000 \mathrm{~g}$ at $4{ }^{\circ} \mathrm{C}$ for $10 \mathrm{~min}$, and serum and plasma were stored at -20 or $-80^{\circ} \mathrm{C}$.

\section{Biochemical analyses}

Whole-blood glucose concentrations were measured on an YSI 2300 STAT Plus ${ }^{\mathrm{TM}}$ (YSI Life Sciences) with an average intra-assay CV of $4.8 \%$ and inter-assay $\mathrm{CV}$ of $5.8 \%$. Plasma insulin and C-peptide were analysed in duplicate using RIA (Millipore) with average intra-assay CV of 7.7 and $4.2 \%$ and inter-assay $\mathrm{CV}$ of 12.6 and $6.4 \%$, respectively. HbA1c and serum high-sensitivity C-reactive protein were measured by the Surrey Pathology Partnership, an accredited laboratory, and serum IL- 6 and TNF- $\alpha$ were measured using a Luminex platform and Biorad bio-plex kits and software (Bio-Rad). Serum TAG, total cholesterol, HDLcholesterol and NEFA were measured on an ILab650 using commercially available kits (Randox Laboratories and Instrumentation Laboratory). All intra-assay $\mathrm{CV}$ were $<2 \%$ and inter-assay $\mathrm{CV} \leq 3 \%$ for lipid measurements. LDL-cholesterol concentration was calculated using the Friedewald formula ${ }^{(27)}$. LPS was measured in duplicate using Endosafe-MCS (Charles River Laboratories), as previously described ${ }^{(15)}$. Serum LPSbinding protein (LBP) and SCD14 concentrations were measured using commercially available kits according to the manufacturer's instructions (Hycult Biotechnology). The average intraassay CV were 3.9 and $8.5 \%$ for LBP and SCD14, respectively.

\section{Amplification and high-throughput sequencing}

Amplification and sequencing were performed as previously described by Ellis et al. ${ }^{(28)}$. Further details are provided in the online Supplementary material.

\section{Bioinformatics}

The sequences were processed in Qiime ${ }^{(29)}$ using the AmpliconNoise $^{(30)}$ pipeline that utilises flowgram information of the sequences to correct for errors. The samples were demultiplexed by exact matching of both barcode and primer, and the sequences were filtered and trimmed on the basis of identification of low-quality signals ${ }^{(31)}$. The filtered flowgrams were clustered to remove platform-specific errors and converted into sequences using the PyroNoise algorithm. The sequences had barcodes and degenerate primers removed before trimming at $400 \mathrm{bp}$. They were then further clustered by SeqNoise to remove PCR single-base errors. In the final step, the Perseus algorithm was used to identify chimeras.

The denoised sequences were classified using the standalone Ribosomal Database Project (RDP) classifier $^{(32)}$. From this, taxa frequencies at five different levels - phylum, class, order, family and genus - were calculated. In addition, a non-supervised approach was used; operational taxonomic units (OTU) were generated at $3 \%$ divergence following pair-wise global sequence alignment and hierarchical clustering with an average linkage algorithm. After generating the abundance tables, multivariate statistical analyses in the context of metadata were done in $\mathrm{R}$ using Vegan package (http://cran.r-project.org/web/ packages/vegan/) for obtaining $\alpha$ - and $\beta$-diversity estimates, as well as permutation ANOVA using distance measures (adonis function). For calculating $\alpha$-diversity measures, the samples were rarefied to the minimum sample size, whereas for other statistics we log-normalised the abundance tables. Where appropriate, $P$-values were adjusted using the BenjaminiHochberg method to control the false discovery rate.

\section{Quantification of bacterial groups by quantitative PCR}

Total bacteria, Bifidobacterium, Roseburia, Lactobacillus, Enterobacteriaceae, Clostridium leptum and Clostridium coccoides groups were quantified using quantitative real-time PCR (qPCR). The qPCR methods are described in the online Supplementary material.

\section{Statistical analysis}

Clinical outcomes and diet data are presented as mean values with their standard errors or medians and interquartile ranges as appropriate. Baseline values between groups were compared using an unpaired $t$ test or Mann-Whitney test and within-group changes with a paired $t$ test or Wilcoxon's matched pairs signedrank test as appropriate. Treatment effects were assessed by comparing differences in changes from baseline between groups using ANCOVA with baseline values as covariates or the Mann-Whitney test if log transformation did not normalise data distribution. AUC for glucose, insulin and C-peptide was calculated using the trapezoid rule. Glucose and insulin data were modelled using Bergman's minimal model (MINMOD Millennium version), as previously described ${ }^{(26)}$. Homoeostasis model assessment (HOMA) for insulin sensitivity (\%S), $\beta$-cell function $(\% \mathrm{~B})$ and insulin resistance (IR) were calculated using the HOMA2 Calculator (http://www.dtu.ox.ac.uk/). Associations between changes in gut bacteria abundance, diet and clinical outcomes were assessed by Kendall's rank correlations. Analysis of qPCR data were performed on $\log _{10}$ transformed values. The level of significance was set at $P<0 \cdot 05$. Data were analysed using GraphPad Prism 6, SPSS versions 21 and 22 and R.

\section{Results}

Fig. 1 shows the flow chart for the study. Of the thirty-two patients recruited, two patients withdrew from the study because of gastrointestinal upset $(n 1)$ and antibiotic treatment ( $n$ 1). Another participant in the prebiotic group was excluded from the data analysis because of antibiotic treatment. Characteristics of the twenty-nine patients who were included in the final data analyses are shown in Table 1 . All patients had been on a stable treatment for at least 3 months before taking part in the study and had no changes to their medications during the study. Two patients in the placebo group did not undergo a full post-supplementation IVGTT because of venous access problems; however, a fasting blood sample was obtained from one of the patients and data from the initial 20 min of the IVGTT for the second patient were included in the data analysis.

Compliance, assessed by the number of unused sachets of supplement, was 96\% (range: 84-100\%) for both treatments. 


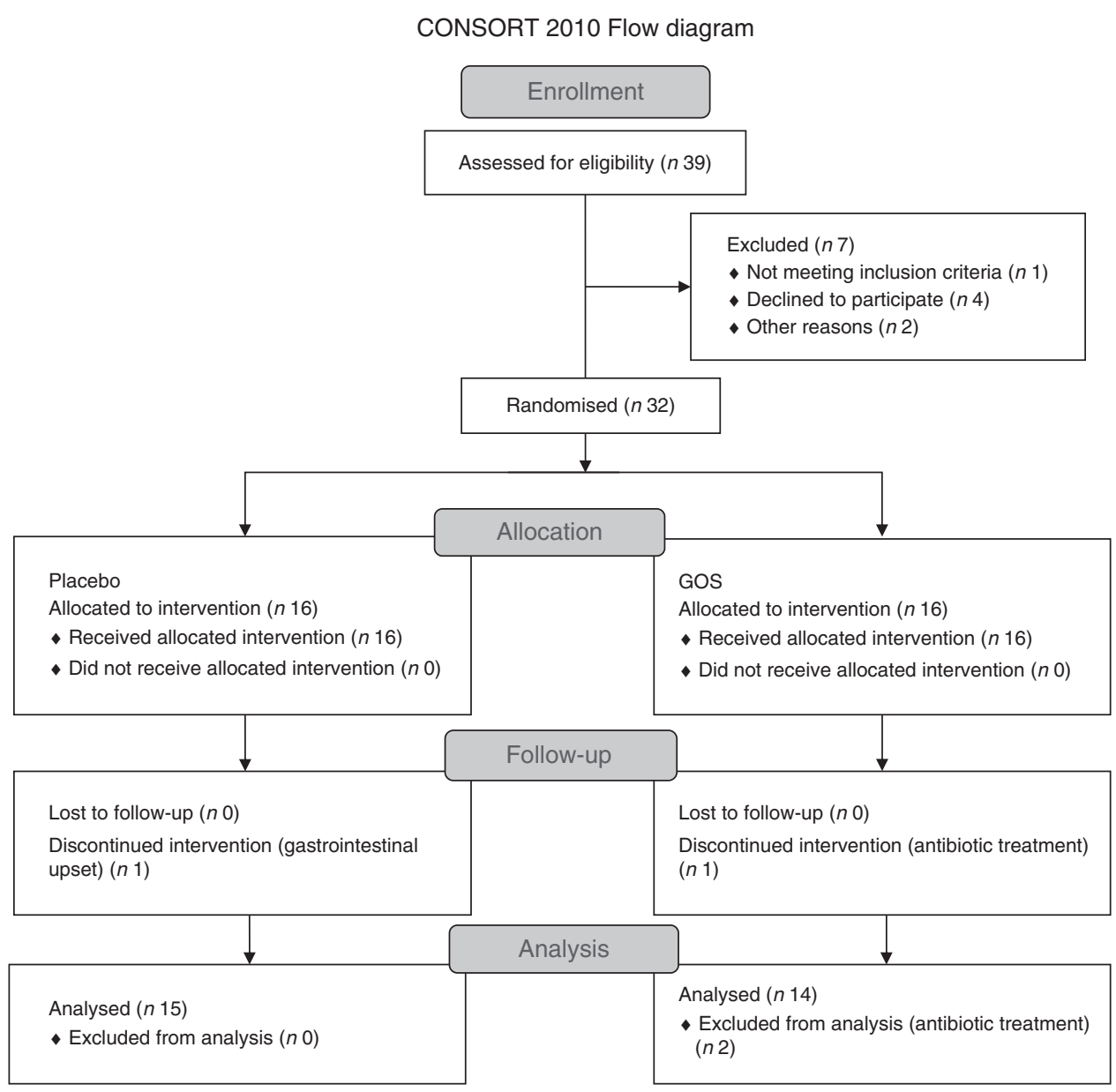

Fig. 1. Flow chart showing the recruitment and retention of patients in the study. GOS, galacto-oligosaccharide.

No adverse side effects were reported by the participants. There were no significant differences between groups in clinical outcomes at baseline; however, Enterobacteriaceae were higher $(P=0.0379)$ (online Supplementary Fig. S2(e)) and Peptostreptococcaceae levels lower $(P=0 \cdot 0019)$ in the prebiotic group at baseline.

\section{Anthropometrics and blood pressure}

Supplementation with the prebiotic fibre had no significant effects on body weight, BMI, body fat percentage, waist circumference or blood pressure when compared with placebo (Table 1).

\section{Intestinal permeability}

Prebiotic supplementation had no significant effect on IP, as measured by urinary recovery of ${ }^{51} \mathrm{Cr}$-EDTA when compared with placebo (Fig. 2).

\section{Glucose tolerance}

Prebiotic treatment had no significant effect on glucose, insulin and C-peptide fasting concentrations or responses during
IVGTT compared with placebo (Table 2). The change in glucose effectiveness at zero insulin in the placebo group was significantly different from the prebiotic group.

\section{Inflammatory markers and lipids}

There were no significant effects of prebiotic treatment on inflammatory markers, LPS or lipids, although the prebiotic tended to reduce total cholesterol and LDL-cholesterol (online Supplementary Table S1).

\section{Dietary assessment}

At baseline, the energy intake in the prebiotic group was 8929 (sEm 538$) \mathrm{kJ} / \mathrm{d}$ with percentage of energy obtained from carbohydrate, sugar, fat, SFA and protein of 42.1 (SEM 2.5), 14.5 (SEM 1.7), 36.6 (SEM 1.5), 12.5 (SEM 0.8) and 15.7 (SEM 0.9)\%, respectively. In the placebo group, the mean daily energy intake was 8683 (SEM 581) kJ and carbohydrate, sugar, fat, SFA and protein provided $40 \cdot 0$ (SEM 1.5) $\%, 14.3$ (SEM 1.0) $\%, 37 \cdot 7$ (SEM $1.5) \%, 12 \cdot 1$ (SEM 0.4 ) $\%$ and $16 \cdot 8$ (SEM 0.8 ) $\%$ of total energy, respectively. The percentage dietary energy from protein increased by $1.1 \%$ in the placebo group, and this was significantly different from that observed in the prebiotic group 
Table 1. Characteristics of the treatment groups at baseline (pre) and after supplementation (post) and diabetes medications* (Mean values with their standard errors; $n 14$ in the prebiotic group and $n 15$ in the placebo group)

\begin{tabular}{|c|c|c|c|c|c|c|c|c|c|}
\hline & \multicolumn{4}{|c|}{ Prebiotic } & \multicolumn{4}{|c|}{ Placebo } & \multirow[b]{3}{*}{$P \dagger$} \\
\hline & \multicolumn{2}{|c|}{ Pre } & \multicolumn{2}{|c|}{ Post } & \multicolumn{2}{|c|}{ Pre } & \multicolumn{2}{|c|}{ Post } & \\
\hline & Mean & SEM & Mean & SEM & Mean & SEM & Mean & SEM & \\
\hline Age (years) & $56 \cdot 7$ & 1.6 & & & $58 \cdot 1$ & 1.7 & & & - \\
\hline Time since diagnosis (years) & 4.6 & 0.6 & & & 4.0 & 0.8 & & & - \\
\hline Ethnicity $(n)$ & & & & & & & & & \\
\hline Caucasian & & & & & & & & & \\
\hline Asian & & & & & & & & & \\
\hline Black & & & & & & & & & \\
\hline Body weight (kg) & $87 \cdot 0$ & 3.5 & 87.6 & 3.6 & $86 \cdot 7$ & 3.2 & $86 \cdot 8$ & 3.2 & 0.335 \\
\hline BMI $\left(\mathrm{kg} / \mathrm{m}^{2}\right)$ & $28 \cdot 0$ & $1 \cdot 1$ & $28 \cdot 2$ & $1 \cdot 1$ & $28 \cdot 4$ & 0.9 & 28.5 & 0.9 & 0.333 \\
\hline Body fat (\%)‡ & $26 \cdot 5$ & $1 \cdot 3$ & $27 \cdot 3 \S$ & $1 \cdot 3$ & $26 \cdot 0$ & 1.5 & 26.5 & 1.4 & 0.514 \\
\hline Waist circumference $(\mathrm{cm}) \|$ & $101 \cdot 3$ & $3 \cdot 1$ & $101 \cdot 7$ & $3 \cdot 6$ & 101.5 & $2 \cdot 7$ & $101 \cdot 2$ & 2.6 & 0.451 \\
\hline Blood pressure (systolic) (mmHg)\| & 136 & 2 & 133 & 3 & 136 & 3 & $132 \S$ & 4 & 0.942 \\
\hline Blood pressure (diastolic) (mmHg)\| & 86 & 2 & 83 & 2 & $84 \cdot 0$ & $1 \cdot 7$ & $81 \cdot 1$ & 1.6 & 0.909 \\
\hline \multicolumn{10}{|l|}{ Diabetes medications $(n) \boldsymbol{\uparrow}$} \\
\hline Metformin & & & & & & & & & \\
\hline Metformin and gliclazide & & & & & & & & & \\
\hline Metformin and sitagliptin & & & & & & & & & \\
\hline Metformin, gliclazide and sitagliptin & & & & & & & & & \\
\hline Metformin, sitagliptin and thiazolidinedione & & & & & & & & & \\
\hline Sitagliptin and gliclazide & & & & & & & & & \\
\hline Gliclazide & & & & & & & & & \\
\hline
\end{tabular}

* There were no differences in baseline (Pre) values between groups $(P>0.05$, unpaired $t$ test).

$\dagger$ The $P$-value is for the comparison of the change between groups with Pre value as covariate (ANCOVA). Other medications $(n)$ used by patients in the prebiotic group were statins (11), blood pressure medication (8), fenofibrate (2), omeprazole (2), low-dose aspirin (1), levothyroxine sodium (1) and citalopram (1). Other medications used in the placebo group were statins (8), blood pressure medication (8), low-dose aspirin (5), Omeprazole (2), benign prostate hyperplasia medications (2), hay fever medication (2), betahistine hydrochloride (1), asthma medication (1), medications for incontinence (2), sleep medication (1) and anti-fungal medication (1).

$\ddagger n 13$ in the placebo group.

$\S$ Significant within-group change $(P<0.05$, paired $t$ test $)$.

II $n 13$ in the prebiotic group.

I The remaining six patients in the placebo group were diet/exercise controlled.

(a)

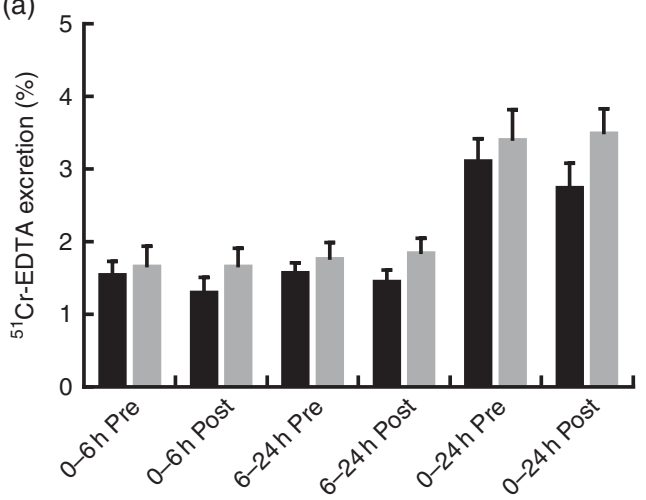

(b)

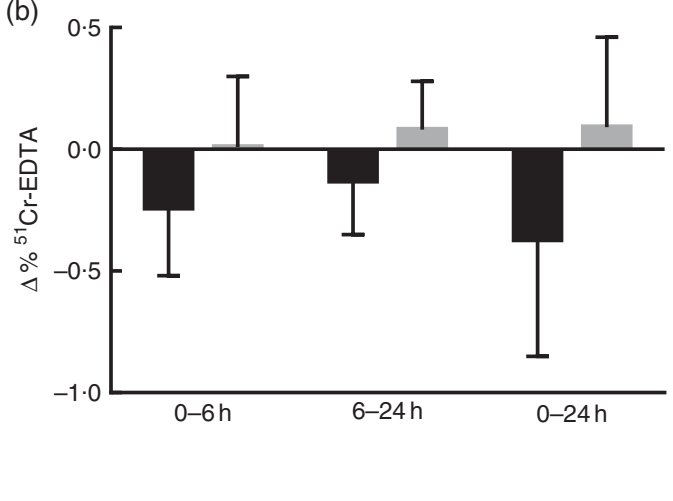

Fig. 2. Intestinal permeability estimated by ${ }^{51} \mathrm{Cr}$-EDTA excreted in urine following 12 weeks of prebiotic ( $\square, n$ 14) or placebo ( $\left.\square, n 15\right)$ supplementation. (a) Percentage ${ }^{51} \mathrm{Cr}$-EDTA excreted before (pre) and after supplementation (post) and (b) change in ${ }^{51} \mathrm{Cr}$-EDTA excreted. Values are means with their standard errors represented by vertical bars. There were no significant differences between treatment groups $(P=0.322, P=0.235$ and $P=0.176$ (ANCOVA) for small intestinal $(0-6 \mathrm{~h})$, colon $(6-24 \mathrm{~h})$ and total tract $(0-24 \mathrm{~h})$ permeability, respectively).

(online Supplementary Table S2). No other significant differences in dietary intakes were observed between groups.

\section{Gut microbiota composition}

Prebiotic fibre treatment did not induce significant changes in diversity, evenness (the relative abundance of species) and richness (the number of species per sample) indices when compared with placebo. However, bacterial diversity, as assessed by the Shannon and inverse Simpson indices, and richness increased significantly within the prebiotic group (online Supplementary Table S3).

Faecal bacterial DNA extraction was unsuccessful (DNA concentration $<50 \mathrm{ng} / \mu \mathrm{l}$ ) for some samples, resulting in $n 11$ in 
Table 2. Glucose tolerance outcomes at baseline and after 12 weeks of supplementation*

(Mean values with their standard errors; medians and interquartile ranges (IQR); $n 13$ for placebo group and $n 14$ for prebiotic group)

\begin{tabular}{|c|c|c|c|c|c|c|c|c|c|}
\hline & \multicolumn{4}{|c|}{ Prebiotic } & \multicolumn{4}{|c|}{ Placebo } & \multirow[b]{3}{*}{$P \dagger$} \\
\hline & \multicolumn{2}{|c|}{ Pre } & \multicolumn{2}{|c|}{ Post } & \multicolumn{2}{|c|}{ Pre } & \multicolumn{2}{|c|}{ Post } & \\
\hline & Mean & SEM & Mean & SEM & Mean & SEM & Mean & SEM & \\
\hline Glucose, fasting $(\mathrm{mmol} / \mathrm{l}) \ddagger$ & $6 \cdot 1$ & 0.4 & $6.8 \S$ & 0.4 & $6 \cdot 2$ & 0.3 & 6.5 & 0.3 & 0.227 \\
\hline Glucose $\mathrm{tAUC}_{180 \min }(\mathrm{mm} \times \mathrm{min})$ & 1319 & 74 & $1414 \S$ & 84 & 1234 & 89 & 1289 & 98 & 0.485 \\
\hline Glucose iAUC $180 \min (\mathrm{mm} \times \mathrm{min})$ & 222 & 33 & 197 & 32 & 153 & 32 & 170 & 35 & 0.221 \\
\hline Insulin, fasting (pmol/l)‡॥\| & 83.5 & $14 \cdot 7$ & 94.0 & $18 \cdot 7$ & $94 \cdot 6$ & $15 \cdot 3$ & $83 \cdot 0$ & $13 \cdot 0$ & 0.543 \\
\hline Insulin tAUC $180 \min (\mathrm{p} \times \min ) \|$ & 6026 & 774 & 7121 & 948 & 6867 & 1091 & 6274 & 821 & 0.112 \\
\hline Insulin $\mathrm{iAUC}_{180 \min }(\mathrm{pm} \times \min )$ & 3522 & 355 & $4301 \S$ & 449 & 3892 & 626 & 3784 & 568 & 0.171 \\
\hline Insulin $\mathrm{tAUC}_{10 \mathrm{~min}}(\mathrm{pm} \times \min ) \boldsymbol{\Phi}$ & 176 & 28 & 175 & 33 & 182 & 33 & 151 & 24 & 0.355 \\
\hline Insulin $\mathrm{i} \mathrm{UC}_{10 \min }(\mathrm{pM} \times \min ) \boldsymbol{\Pi}$ & 37 & 14 & 18 & 6 & 23 & 19 & 16 & 12 & 0.946 \\
\hline C-peptide $\mathrm{AUCC}_{180 \min }(\mathrm{pm} \times \min )$ & 339 & 30 & 403 & 41 & 342 & 41 & 333 & 44 & 0.166 \\
\hline C-peptide $\mathrm{iAUC}_{180 \min }(\mathrm{pm} \times \min )$ & 71 & 9 & 94 & 11 & 73 & 14 & 59 & 20 & 0.111 \\
\hline $\mathrm{HbA} 1 \mathrm{c}(\mathrm{mmol} / \mathrm{mol}) \ddagger \|$ & $51 \cdot 2$ & $3 \cdot 1$ & $53 \cdot 1$ & $3 \cdot 2$ & $46 \cdot 3$ & 1.8 & $48 \cdot 4$ & 2.4 & 0.946 \\
\hline $\mathrm{HbA1c}(\%) \ddagger$ & $6 \cdot 8$ & 0.3 & $7 \cdot 0$ & 0.3 & $6 \cdot 4$ & 0.2 & $6 \cdot 6$ & 0.2 & - \\
\hline AIRg (mU/l per min) & $39 \cdot 1$ & $13 \cdot 4$ & $21 \cdot 2$ & $5 \cdot 2$ & $38 \cdot 3$ & $15 \cdot 6$ & $23 \cdot 1$ & $10 \cdot 5$ & 0.856 \\
\hline DI & \multirow{2}{*}{\multicolumn{2}{|c|}{38}} & & & & & & & 0.4507 \\
\hline Median & & & \multicolumn{2}{|c|}{$49 \cdot 8$} & \multicolumn{2}{|c|}{53.6} & \multicolumn{2}{|c|}{$20 \cdot 6$} & \\
\hline IQR & \multicolumn{2}{|c|}{$5 \cdot 5-119.1$} & \multicolumn{2}{|c|}{$2 \cdot 7-111 \cdot 3$} & \multirow{2}{*}{\multicolumn{2}{|c|}{$0-172 \cdot 4$}} & \multicolumn{2}{|c|}{$0 \cdot 1-36 \cdot 8$} & \\
\hline $\mathrm{SI}\left((\mathrm{mU} / \mathrm{l})^{-1} \mathrm{~min}^{-1}\right)$ & \multirow{2}{*}{\multicolumn{2}{|c|}{1.95}} & \multirow{2}{*}{\multicolumn{2}{|c|}{$2 \cdot 18$}} & & & & & 0.2358 \\
\hline Median & & & & & \multicolumn{2}{|c|}{4.48} & \multicolumn{2}{|c|}{1.91} & \\
\hline IQR & \multicolumn{2}{|c|}{$0.95-3.98$} & \multicolumn{2}{|c|}{$0.16-4.32$} & \multicolumn{2}{|c|}{$1 \cdot 31-172 \cdot 5$} & \multicolumn{2}{|c|}{$0.22-4.84$} & \\
\hline GEZI (per min) & \multirow{2}{*}{\multicolumn{2}{|c|}{0.022}} & \multirow{2}{*}{\multicolumn{2}{|c|}{0.018}} & & & & 0.0212 \\
\hline Median & & & & & & & \multicolumn{2}{|c|}{0.020} & \\
\hline IQR & 0.01 & 025 & 0.00 & & -0.2 & 0.021 & 0.01 & & \\
\hline$\beta$-Cell function $(\mathrm{mU} / \mathrm{mm})$ & $173 \cdot 1$ & $30 \cdot 4$ & 139.5 & $24 \cdot 8$ & $165 \cdot 9$ & $26 \cdot 7$ & $113 \cdot 2 \S$ & 14.6 & 0.350 \\
\hline $\mathrm{IR}\left(\mathrm{mmol} \mathrm{mU} / \mathrm{I}^{2}\right)$ & 3.6 & 0.8 & $4 \cdot 3$ & 0.9 & $4 \cdot 3$ & 0.8 & 3.9 & 0.9 & 0.337 \\
\hline HOMA2 \%Bł & $100 \cdot 4$ & $10 \cdot 9$ & $90 \cdot 2$ & $11 \cdot 6$ & $100 \cdot 2$ & $10 \cdot 9$ & $81 \cdot 3 \S$ & $7 \cdot 0$ & 0.362 \\
\hline HOMA2 \%S‡ & & & & & & & & & 0.2147 \\
\hline Median & & & & & & & & & \\
\hline IQR & $46 \cdot($ & & 37. & & & & 39. & & \\
\hline HOMA2 IR & & & & & & & & & 0.1994 \\
\hline Median & & & & & & & & & \\
\hline IQR & 1.0 & & 1.0 & & & & $1 \cdot 2$ & & \\
\hline
\end{tabular}

tAUC, total AUC; iAUC, incremental AUC; AIRg: acute insulin response to glucose; DI, disposition index; SI, insulin sensitivity; GEZI, glucose effectiveness at zero insulin; IR, insulin resistance; HOMA, homoeostasis model assessment; \%B, \% $\beta$-cells; \%S, \% sensitivity.

* There were no differences in baseline (Pre) values between groups $(P>0.05$, unpaired $t$ test or Mann-Whitney test)

$\dagger$ The $P$-value is for the comparison of the change between groups with Pre value as covariate (ANCOVA).

$\ddagger n 15$ for placebo group.

$\S$ Significant within-group change $(P<0.05$, paired $t$ test or Wilcoxon's matched pairs signed-rank test).

॥ ANCOVA performed on log-transformed values.

II $n 14$ for placebo group.

the prebiotic group and $n 12$ in the placebo group for the qPCR data set. After removing samples with $<400 \mathrm{bp}$, the metagenomics data set consisted of $n 7$ in the prebiotic group and $n 9$ in the placebo group.

Consistent with previous reports on composition of the gut microbiota in humans, Bacteroidetes and Firmicutes were the two dominant phyla followed by Proteobacteria, unclassified bacteria and Actinobacteria (data not shown). Bacterial community structure in the treatment groups changed only slightly during the study, but the change was greater in the prebiotic group, as can be observed in the non-metric multidimensional scaling (NMDS) plot (online Supplementary Fig. S1(A)). The change in the placebo group was mainly because of the changes in metformin-treated patients (online Supplementary Fig. S1(B)). However, comparison of bacteria abundances at all taxonomic levels did not reveal any significant effect of treatment when adjusted for multiple testing (data not shown). Nonetheless, permutation ANOVA showed a trend towards an effect of treatment $(P=0.099)$ at the OTU level. When metformin was included as a cofactor, metformin had a significant effect on bacterial community structure at the genus level $\left(R^{2}=0.084, P=0.009\right)$, whereas only a trend was detected when the analysis was performed on OTU $\left(R^{2}=0 \cdot 039, P=0 \cdot 078\right)$.

\section{Quantification of bacterial groups by quantitative real-time $P C R$}

Prebiotic treatment had no significant effect on Bifidobacterium or any of the other bacteria measured (online Supplementary Fig. S2). Bifidobacterium levels increased in both groups; however, the change within the prebiotic group was greater and close to significance $(P=0 \cdot 0582)$.

\section{Correlations between changes in bacteria, clinical outcomes and dietary intakes}

As an a priori aim was to investigate the role of prebiotic fibre intake specifically for hypothesis generation, correlations were 
calculated for each treatment group separately. The correlations differed between the two groups as can be observed from the different patterns in the heat maps (online Supplementary Fig. S3(A-E)). Changes in large bowel permeability $\left({ }^{51} \mathrm{Cr}-\mathrm{EDTA}\right.$ 6-24h excretion) were positively correlated with bacterial changes at all taxonomic levels in the prebiotic group. The strongest correlations were for Verrucomicrobia, Euryarchaeota and Methanobacteria (online Supplementary Fig. S3(A, B)); Rikenellaceae and unclassified Clostridiales (online Supplementary Fig. S3(D)); and six genera, including Alistipes, Shigella and Flavonifractor (online Supplementary Fig. S3(E)). Furthermore, changes in small intestinal and total IP $\left({ }^{51} \mathrm{Cr}\right.$-EDTA $0-6 \mathrm{~h}$ and $0-24 \mathrm{~h}$ excretion, respectively) correlated positively with changes in Enterobacteriaceae measured by qPCR $(r 0.527$, $P=0.024$, adjusted $P=0.51$ for both small intestinal and total tract permeability) in the prebiotic group. In contrast, only few bacteria correlated with changes in glucose tolerance outcomes; Actinobacteria and Bifidobacterium correlated positively and Veillonellaceae and Clostridium cluster XVIII inversely with glucose total AUC (tAUC) (online Supplementary Fig. S3(A-D)). Unclassified Enterobacteriaceae correlated positively with fasting glucose, insulin sensitivity, high-sensitivity C-reactive protein and waist circumference (online Supplementary Fig. S3(D)).

In the prebiotic group the strongest correlations between bacteria and inflammatory markers were observed for sCD14, which correlated inversely with Verrucomicrobia and unclassified bacteria, Erysipelotrichales and Verrucomicrobiales, Verrucomicrobiacea, Lactobacillaceae and Erysipelotrichaceae (online Supplementary Fig. S3(A, C, D)). Actinobacteria and Firmicutes correlated positively with IL- 6 and TNF- $\alpha$, respectively (online Supplementary Fig. S3(A)). Furthermore, IL-6 correlated positively with Bifidobacterium and negatively with Veillonellaceae and Dialister (online Supplementary Fig. S3(C, D, E)). Changes in small IP correlated with glucose response (incremental AUC) and carbohydrate energy percentage $(r-0.429, P=0.033$ for both) and colon IP correlated with protein intake $(r 0.464, P=0.021)$ in the prebiotic group. However, because of the small sample size, apart from the association between Veillonellaceae and IL- 6 and glucose tAUC ( $r-0.90$, adjusted $P=0.042$ for both) none of these correlations in the prebiotic group were statistically significant after adjustment for multiple testing.

\section{Discussion}

In this study, 12 weeks of prebiotic fibre supplementation did not have a significant beneficial effect on glucose tolerance outcomes in individuals with well-controlled T2D. Although there was a decrease in the IP in the prebiotic group, this was not statistically significant. Because of the number of patients presenting with permeability values within the normal range being higher than expected based on our previous work $(50 v .28 \%)^{(3)}$, in future, it would be deemed necessary to test the role of prebiotics in those with a demonstrated impairment in barrier function to assess the true functionality of this dietary fibre.

Bifidobacterium levels increased in both treatment groups, although there was a trend towards post-intervention levels being higher in the prebiotic group. GOS has previously been shown to increase bifidobacteria levels, although it was noted that some volunteers were non-responders ${ }^{(23,24,33,34)}$ and one study did not find a significant bifidogenic effect of GOS compared with placebo treatment ${ }^{(35)}$. Interestingly, others have reported a poorer bifidogenic effect of GOS in males and overweight individuals ${ }^{(34)}$. However, other factors may play a role in these negative findings including the type and dosage of GOS administered, background diet, as well as the methods of analysis of Bifidobacterium ${ }^{(33)}$. As for the background diet, particularly the relatively high dietary fibre intake $(>20 \mathrm{~g} / \mathrm{d})$ in this cohort may have diminished the effect of the prebiotic supplement.

We used a dose of $5.5 \mathrm{~g}$ prebiotic/d, which may be considered to be low compared with other studies in which doses of $10 \mathrm{~g}$ or more prebiotic were consumed ${ }^{(16,18,19)}$. A duration of 12 weeks may not have been sufficient to elicit a significant effect on clinical outcomes, although it would have been ample time for changes in the microbiota to become apparent. Resistant starch (which is also a prebiotic) improves first-phase insulin secretion and insulin sensitivity in individuals at risk of T2D within this time scale ${ }^{(26,36)}$; however, it shows less efficacy in those already with $\mathrm{T}_{2} \mathrm{D}^{(37)}$. An unexpected finding was a decrease in first-phase insulin secretion and an increase in HbA1c in both groups in addition to an increase in fasting glucose within the prebiotic group. This suggests that short-term treatment with a low-dose prebiotic fibre does not prevent further deterioration of key clinical parameters in T2D. The metabolic derangements in established T2D may be difficult to reverse, as shown by the fact that prebiotic supplementation $^{(18,19,37)}$ does not improve glucose control in T2D, whereas a high efficacy is shown in metabolic syndrome.

Metformin had a significant effect on the intestinal bacterial composition at the genus level, although it only explained a small part $(<10 \%)$ of the variation in bacterial composition. Others have recently demonstrated a profound effect of metformin on intestinal bacterial community, bile acids, gut architecture, intestinal glucose utilisation, as well as circulating glucagon-like peptide $1, \operatorname{LBP}$ and $\operatorname{LPS}^{(9,38-43)}$. The effect of metformin on glucose control may partly be mediated by these intestinal effects; the increase in the mucin-degrading bacteria Akkermansia muciniphila following metformin treatment is thought to be beneficial ${ }^{(15,40)}$. Prebiotics have been shown to increase A. muciniphila in mice ${ }^{(15)}$; however, we did not observe significant changes in A. muciniphila levels following prebiotic treatment. However, it is a limitation of this study that all thirteen participants for whom bacterial data were available in the prebiotic group were on metformin, whereas only seven participants in the placebo group were on metformin. It seems plausible that metformin may have masked the effects of the prebiotic in the present study, and it is a possible explanation underlying the discrepancy with both animal work and metabolic syndrome, as metformin treatment would not be administered in animal models of T2D.

The fact that the cohort in this study consisted of patients with well-controlled T2D may also play a role. Inflammatory markers were generally low in this group, and this may have been because of a favourable combination of lifestyle factors and 
medication. However, inflammatory markers are often low in patients with T2D. This may be because some of the antihypertensive and lipid-lowering medications taken by the patients in this study have anti-inflammatory properties and these types of medications may also influence gut bacterial composition $^{(44)}$. No clear links between IP and intestinal bacteria were found in this study. The positive correlation between Enterobacteriaceae and ${ }^{51}$ Cr-EDTA recovery was not significant after adjustment for multiple testing, although it has been useful in hypothesis generation for future work. Others have suggested that a potential link exists between gut health and Enterobacteriaceae because of endotoxin-producing opportunistic pathogens in this bacterial family ${ }^{(45)}$. Nevertheless, we found a significant inverse association between changes in Veillonellaceae and IL-6 and glucose tAUC, suggesting a link between this bacterial family, inflammation and glucose response. Veillonellaceae comprises several acetate and propionate producers $^{(46)}$, and it has been suggested that SCFA may mediate some of the beneficial effects of prebiotics on host metabolism ${ }^{(47)}$. The limitations in this study are primarily related to the small sample size, which makes it difficult to detect subtle effects of a low dose of prebiotic in a heterogeneous study cohort and the potential confounding effects of various medications. In this study, a decision was made at the outset to include numerous clinical and bacterial outcomes, in order to be hypothesis generating for future more focused clinical studies.

In conclusion, supplementation with a low-dose prebiotic for 12 weeks in metformin-treated T2D patients did not improve glucose control; this is now in line with other work showing lack of efficacy of dietary fibres in the treatment of T2D in contrast to their beneficial role in T2D prevention ${ }^{(37)}$. However, our study was limited by the small sample size. Before adjustment for multiple testing, many significant associations between changes in intestinal bacteria and clinical outcomes were observed during this study, providing focus and avenues for further work. The commonly used drug metformin is now known to be a significant confounder in the study of bacterial populations in $\mathrm{T} 2 \mathrm{D}$ and must be accounted for in future work in this cohort.

\section{Acknowledgements}

The authors acknowledge the staff at the CEDAR Centre, Royal Surrey County Hospital, and Dr Caroline Bodinham and Dr Martin Whyte, University of Surrey, for their assistance in the clinical experiments, Amandine Bever, Catholic University of Louvain, for assistance in ELISA and to the study participants for their time and support. The authors also acknowledge Clasado Ltd for providing the supplements.

This study was funded by an EFSD clinical research grant. The research was supported by the National Institute for Health Research Clinical Research Network: Kent, Surrey and Sussex.

M. D. R.: obtained the funding, designed and supervised the research. C. P., E. G., F. H., P. H. and M. D. R.: conducted the clinical experiments. R. J. E.: performed the next-generation sequencing. E. J.: performed the DNA extraction. O. D.: performed qPCR. T. D. and P. D. C.: performed inflammatory marker and LPS measurements. C. P., U. Z. I. and H. W.: analysed the data. J. W.: provided medical supervision. C. P., U. Z. I., R. J. E., O. D. and M. D. R.: wrote the manuscript. R. L. R., G. R. G., O. D. and P. D. C.: edited the manuscript. M. D. R. is the guarantor of this work and, as such, had full access to all the data in the study and takes responsibility for the integrity of the data and the accuracy of the data analysis.

The authors declare that there is no duality of interest associated with this manuscript.

\section{Supplementary material}

For supplementary material/s referred to in this article, please visit https://doi.org/10.1017/S0007114516004086

\section{References}

1. Cani PD, Amar J, Iglesias MA, et al. (2007) Metabolic endotoxemia initiates obesity and insulin resistance. Diabetes $\mathbf{5 6}$, $1761-1772$.

2. Cani PD, Bibiloni R, Knauf C, et al. (2008) Changes in gut microbiota control metabolic endotoxemia-induced inflammation in high-fat diet-induced obesity and diabetes in mice. Diabetes 57, 1470-1481.

3. Horton F, Wright J, Smith L, et al. (2014) Increased intestinal permeability to oral chromium $\left({ }^{51} \mathrm{Cr}\right)$-EDTA in human type 2 diabetes. Diabet Med 31, 559-563.

4. Creely SJ, McTernan PG, Kusminski CM, et al. (2007) Lipopolysaccharide activates an innate immune system response in human adipose tissue in obesity and type 2 diabetes. Am J Physiol Endocrinol Metab 292, E740-E747.

5. Pussinen PJ, Havulinna AS, Lehto M, et al. (2011) Endotoxemia is associated with an increased risk of incident diabetes. Diabetes Care 34, 392-397.

6. Ghoshal S, Witta J, Zhong J, et al. (2009) Chylomicrons promote intestinal absorption of lipopolysaccharides. J Lipid Res 50, 90-97.

7. Larsen N, Vogensen FK, van den Berg FW, et al. (2010) Gut microbiota in human adults with type 2 diabetes differs from non-diabetic adults. PLOS ONE 5, e9085.

8. Wu X, Ma C, Han L, et al. (2010) Molecular characterisation of the faecal microbiota in patients with type II diabetes. Curr Microbiol 61, d69-d78.

9. Karlsson FH, Tremaroli V, Nookaew I, et al. (2013) Gut metagenome in European women with normal, impaired and diabetic glucose control. Nature 498, 99-103.

10. Remely M, Aumueller E, Jahn D, et al. (2014) Microbiota and epigenetic regulation of inflammatory mediators in type 2 diabetes and obesity. Benef Microbes 5, 33-43.

11. Sato J, Kanazawa A, Ikeda F, et al. (2014) Gut dysbiosis and detection of 'live gut bacteria' in blood of Japanese patients with type 2 diabetes. Diabetes Care 37, 2343-2350.

12. Lambeth SM, Carson T, Lowe J, et al. (2015) Composition, diversity and abundance of gut microbiome in prediabetes and type 2 diabetes. J Diabetes Obes 2, 1-7.

13. Mattace Raso G, Simeoli R, Iacono A, et al. (2014) Effects of a Lactobacillus paracasei B21060 based synbiotic on steatosis, insulin signaling and toll-like receptor expression in rats fed a high-fat diet. J Nutr Biochem 25, 81-90.

14. Wang Z, Xiao G, Yao Y, et al. (2006) The role of bifidobacteria in gut barrier function after thermal injury in rats. $J$ Trauma 61, 650-657. 
15. Everard A, Belzer C, Geurts L, et al. (2013) Cross-talk between Akkermansia muciniphila and intestinal epithelium controls diet-induced obesity. Proc Natl Acad Sci 110, 9066-9071.

16. Pourghassem Gargari B, Dehghan P, Aliasgharzadeh A, et al. (2013) Effects of high performance inulin supplementation on glycemic control and antioxidant status in women with type 2 diabetes. Diabetes Metab J 37, 140-148.

17. Yamashita K, Kawai K \& Itakura M (1984) Effects of fructooligosaccharides on blood glucose and serum lipids in diabetic subjects. Nutr Res 4, 961-966.

18. Alles MS, de Roos NM, Bakx JC, et al. (1999) Consumption of fructooligosaccharides does not favorably affect blood glucose and serum lipid concentrations in patients with type 2 diabetes. Am J Clin Nutr 69, 64-69.

19. Luo J, Van Yperselle M, Rizkalla SW, et al. (2000) Chronic consumption of short-chain fructooligosaccharides does not affect basal hepatic glucose production or insulin resistance in type 2 diabetics. J Nutr 130, 1572-1577.

20. Aliasgharzadeh A, Dehghan P, Gargari BP, et al. (2015) Resistant dextrin, as a prebiotic, improves insulin resistance and inflammation in women with type 2 diabetes: a randomised controlled clinical trial. BrJ Nutr 113, 321-330.

21. Gargari BP, Namazi N, Khalili M, et al. (2015) Is there any place for resistant starch, as alimentary prebiotic, for patients with type 2 diabetes? Complement Ther Med 23, 810-815.

22. Dehghan P, Farhangi MA, Tavakoli F, et al. (2016) Impact of prebiotic supplementation on T-cell subsets and their related cytokines, anthropometric features and blood pressure in patients with type 2 diabetes mellitus: a randomized placebocontrolled Trial. Complement Ther Med 24, 96-102.

23. Vulevic J, Juric A, Walton GE, et al. (2015) Influence of galacto-oligosaccharide mixture (B-GOS) on gut microbiota, immune parameters and metabonomics in elderly persons. $\mathrm{Br}$ J Nutr 114, 586-595.

24. Davis LM, Martinez I, Walter J, et al. (2011) Barcoded pyrosequencing reveals that consumption of galactooligosaccharides results in a highly specific bifidogenic response in humans. PlOS ONE 6, e25200.

25. Teshima CW \& Meddings JB (2008) The measurement and clinical significance of intestinal permeability. Curr Gastroenterol Rep 10, 443-449.

26. Bodinham CL, Smith L, Wright J, et al. (2012) Dietary fibre improves first-phase insulin secretion in overweight individuals. PLOS ONE 7, e40834.

27. Friedewald WT, Levy RI \& Fredrickson DS (1972) Estimation of the concentration of low-density lipoprotein cholesterol in plasma, without use of the preparative ultracentrifuge. Clin Chem 18, 499-502.

28. Ellis RJ, Bruce KD, Jenkins C, et al. (2013) Comparison of the distal gut microbiota from people and animals in Africa. PLOS ONE 8, e 54783.

29. Caporaso JG, Kuczynski J, Stombaugh J, et al. (2010) QIIME allows analysis of high-throughput community sequencing data. Nat Methods 7, 335-336.

30. Quince C, Lanzen A, Davenport RJ, et al. (2011) Removing noise from pyrosequenced amplicons. BMC Bioinformatics 12, 38 .
31. Quince C, Lanzén A, Curtis TP, et al. (2009) Accurate determination of microbial diversity from 454 pyrosequencing data. Nat Methods 6, 639-641.

32. Wang Q, Garrity GM, Tiedje JM, et al. (2007) Naive Bayesian classifier for rapid assignment of rRNA sequences into the new bacterial taxonomy. Appl Environ Microbiol $\mathbf{7 3}$, 5261-5267.

33. Davis LM, Martinez I, Walter J, et al. (2010) A dose dependent impact of prebiotic galactooligosaccharides on the intestinal microbiota of healthy adults. Int $J$ Food Microbiol 144, 285-292.

34. Walton GE, van den Heuvel EG, Kosters MH, et al. (2012) A randomised crossover study investigating the effects of galacto-oligosaccharides on the faecal microbiota in men and women over 50 years of age. Br J Nutr 107, 1466-1475.

35. Alles MS, Hartemink R, Meyboom S, et al. (1999) Effect of transgalactooligosaccharides on the composition of the human intestinal microflora and on putative risk markers for colon cancer. Am J Clin Nutr 69, 980-991.

36. Johnston KL, Thomas EL, Bell JD, et al. (2010) Resistant starch improves insulin sensitivity in metabolic syndrome. Diabet Med 27, 391-397.

37. Bodinham CL, Smith L, Thomas EL, et al. (2014) Efficacy of increased resistant starch consumption in human type 2 diabetes. Endocr Connect 3, 75-84.

38. Napolitano A, Miller S, Nicholls AW, et al. (2014) Novel gutbased pharmacology of metformin in patients with type 2 diabetes mellitus. PLOS ONE 9, e100778.

39. Lee H \& Ko G (2014) Effect of metformin on metabolic improvement and the gut microbiota. Appl Environ Microbiol 80, 5935-5943.

40. Shin NR, Lee JC, Lee HY, et al. (2014) An increase in the Akkermansia spp. population induced by metformin treatment improves glucose homeostasis in diet-induced obese mice. Gut $\mathbf{6 3}, 727-735$.

41. Mithieux G, Rajas F \& Zitoun C (2006) Glucose utilization is suppressed in the gut of insulin-resistant high fat-fed rats and is restored by metformin. Biochem Pharmacol 72, $1757-1762$

42. Moreno-Navarrete JM, Ortega F, Serino M, et al. (2012) Circulating lipopolysaccharide-binding protein (LBP) as a marker of obesity-related insulin resistance. Int $J$ Obes $\mathbf{3 6}$, 1442-1449.

43. Forslund K, Hildebrand F, Nielsen T, et al. (2015) Disentangling type 2 diabetes and metformin treatment signatures in the human gut microbiota. Nature 528, 262-266.

44. Catry E, Pachikian BD, Salazar N, et al. (2015) Ezetimibe and simvastatin modulate gut microbiota and expression of genes related to cholesterol metabolism. Life Sci 132, 77-84.

45. Walker AW \& Lawley TD (2012) Therapeutic modulation of intestinal dysbiosis. Pharmacol Res 69, 75-86.

46. Kettle H, Louis P, Holtrop G, et al. (2015) Modelling the emergent dynamics and major metabolites of the human colonic microbiota. Environ Microbiol 17, 1615-1630.

47. Byrne CS, Chambers ES, Morrison DJ, et al. (2015) The role of short chain fatty acids in appetite regulation and energy homeostasis. Int J Obes (Lond) 39, 1331-1338. 